\title{
ON THE APPROXIMATION OF ONE CLASS OF IMPULSE FUNCTIONS
}

\author{
OLGA RAHNEVA ${ }^{1}$, HRISTO KISKINOV ${ }^{2}$, HRISTO MELEMOV ${ }^{3}$, AND \\ MARTIN STIEGER ${ }^{4}$ \\ ${ }^{1}$ Faculty of Economy and Social Sciences \\ University of Plovdiv Paisii Hilendarski \\ 24, Tzar Asen Str., 4000 Plovdiv, BULGARIA \\ ${ }^{2}$ Faculty of Mathematics and Informatics \\ University of Plovdiv Paisii Hilendarski \\ 24, Tzar Asen Str., 4000 Plovdiv, BULGARIA \\ ${ }^{3}$ University of Plovdiv Paisii Hilendarski \\ Branch Smolyan \\ 32 Dicho Petrov Str., 4700 Smolyan, BULGARIA \\ ${ }^{4}$ Department of Business Administration \\ Allensbach University \\ Lohnerhofstrasse 2, 78467 Konstanz, GERMANY
}

\begin{abstract}
In this paper we consider a new class of cumulative distribution functions belonging to the important class of functions arising from the theory of impulse techniques, neural networks and debugging theory.

By this new family of cumulative functions we study the Hausdorff approximation of the impulse function $\sigma^{* *}(t)$.

Numerical examples, illustrating our results using the programming environment CAS MATHEMATICA are presented.
\end{abstract}

AMS Subject Classification: $41 \mathrm{~A} 46$

Key Words: impulse function $\sigma^{* *}(t)$, cumulative distribution function, emitting chart, Hausdorff approximation, upper and lower bounds

Received: June 11, 2018;

Published: September 15, 2018.
Accepted: September 1, 2018;

doi: $\quad 10.12732 /$ npsc.v26i3.1 


\section{INTRODUCTION}

Based on some extensions of the Chen and Poisson-exponential cumulative distribution functions [1]-[12], in [13] we studied the following cumulative distribution function belonging to the important class of functions arising from the theory of impulse techniques, debugging theory, population dynamics and cell growth models:

$$
N(t)=\frac{e^{\lambda e^{-\beta t^{m}}}-e^{\lambda}}{1-e^{\lambda}}
$$

with

$$
t_{0}=\left(-\frac{1}{\beta} \ln \left(\frac{1}{\lambda} \ln \left(\frac{1+e^{\lambda}}{2}\right)\right)\right)^{\frac{1}{m}} ; \quad N\left(t_{0}\right)=\frac{1}{2},
$$

where $\beta, \lambda>0$ and $m$ is an even number.

In this paper we consider the following new cumulative function with application to the theory of impulse techniques:

$$
M(t)=\left(\frac{e^{\lambda e^{-\beta t^{m}}}-e^{\lambda}}{1-e^{\lambda}}\right)^{\alpha}
$$

with

$$
t_{0}=\left(-\frac{1}{\beta} \ln \left(\frac{1}{\lambda} \ln \left(e^{\lambda}+\frac{1-e^{\lambda}}{2^{\frac{1}{\alpha}}}\right)\right)\right)^{\frac{1}{m}} ; \quad M\left(t_{0}\right)=\frac{1}{2},
$$

where $\beta, \lambda, \alpha>0$ and $m$ is an even number.

The typical example of an impulse function from antenna feeder technique has the following shape (see, Fig. 1):

$$
\sigma^{* *}(t)=\left\{\begin{array}{l}
1, t \in[-\infty,-1) \cup(1,+\infty) \\
0, t \in[-1,1]
\end{array}\right.
$$

In this paper we study the Hausdorff approximation of the impulse function $\sigma^{* *}(t)$ (see Definition 1) by the family (3)-(4).

Furthermore, we propose a software module (intellectual property) within the programming environment CAS Mathematica for the analysis. The models have been tested with real-world data.

\section{HAUSDORFF APPROXIMATION OF THE IMPULSE FUNCTION $\sigma^{* *}(T)$}

Definition 1. [14] The Hausdorff distance (the H-distance) $\rho(f, g)$ between two interval functions $f, g$ on $\Omega \subseteq \mathbb{R}$, is the distance between their completed graphs $F(f)$ 


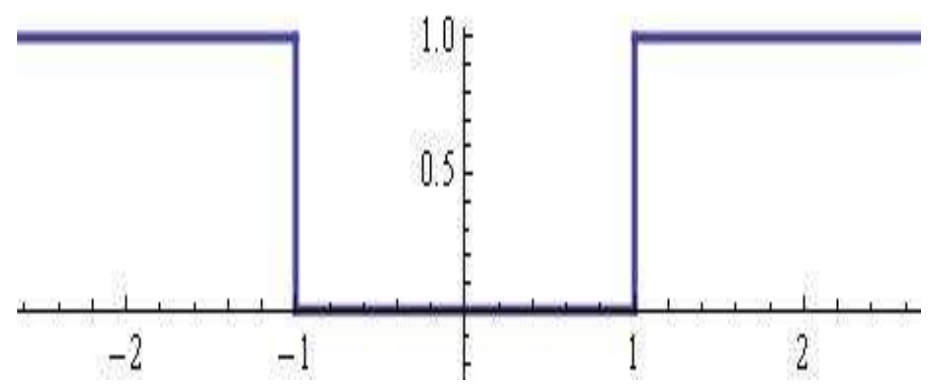

Figure 1: The signal of $\sigma^{* *}(t)-$ type.

and $F(g)$ considered as closed subsets of $\Omega \times \mathbb{R}$. More precisely,

$$
\rho(f, g)=\max \left\{\sup _{A \in F(f)} \inf _{B \in F(g)}\|A-B\|, \sup _{B \in F(g)} \inf _{A \in F(f)}\|A-B\|\right\},
$$

wherein $\|$.$\| is any norm in \mathbb{R}^{2}$, e. g. the maximum norm $\|(t, x)\|=\max \{|t|,|x|\}$; hence the distance between the points $A=\left(t_{A}, x_{A}\right), B=\left(t_{B}, x_{B}\right)$ in $\mathbb{R}^{2}$ is $\|A-B\|=$ $\max \left(\left|t_{A}-t_{B}\right|,\left|x_{A}-x_{B}\right|\right)$.

The one-sided Hausdorff distance $d$ between the impulse function $\sigma^{* *}(t)$ and the function $((3)-(4))$ satisfies the relation

$$
M\left(t_{0}-d\right)=d
$$

The following theorem gives upper and lower bounds for $d$

Theorem 2. Let

$$
\begin{gathered}
p=\frac{1}{2}, \\
q=-1+\frac{m \beta \alpha 2^{\frac{1}{\alpha}}\left(e^{\lambda}+\frac{1-e^{\lambda}}{2^{\frac{1}{\alpha}}}\right) \ln \left(e^{\lambda}+\frac{1-e^{\lambda}}{2^{\frac{1}{\alpha}}}\right)}{2\left(1-e^{\lambda}\right)}\left(-\frac{1}{\beta} \ln \left(\frac{1}{\lambda} \ln \left(e^{\lambda}+\frac{1-e^{\lambda}}{2^{\frac{1}{\alpha}}}\right)\right)\right)^{\frac{m-1}{m}} \\
r=-2.1 q>0 .
\end{gathered}
$$

For the one-sided Hausdorff distance $d$ between $\sigma^{* *}(t)$ and the function ((3)-(4)) for

$$
r>e^{1.05} \approx 1.36079
$$

the following inequalities hold:

$$
d_{l}=\frac{1}{r}<d<\frac{\ln r}{r}=d_{r}
$$




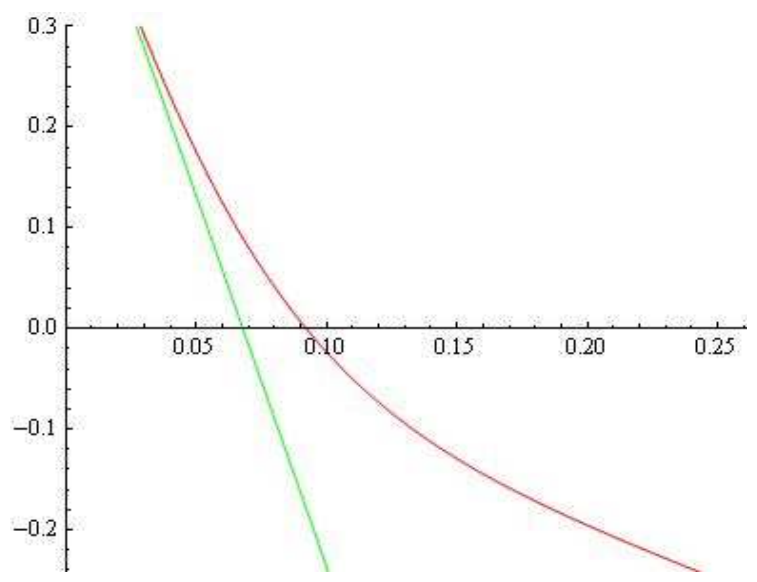

Figure 2: The functions $F(d)$ and $G(d)$ for $\beta=20 ; \lambda=0.7 ; m=12 ; \alpha=1.5$.

Proof. Let us examine the function:

$$
F(d)=M\left(t_{0}-d\right)-d
$$

From $F^{\prime}(d)<0$ we conclude that the function $F$ is decreasing.

Consider the function

$$
G(d)=p+q d
$$

From the Taylor expansion we obtain $G(d)-F(d)=O\left(d^{2}\right)$.

Hence $G(d)$ approximates $F(d)$ with $d \rightarrow 0$ as $O\left(d^{2}\right)$ (see Fig. 2).

In addition $G^{\prime}(d)<0$.

Further, for $r>e^{1.05}$ we have $G\left(d_{l}\right)>0$ and $G\left(d_{r}\right)<0$.

This completes the proof of the theorem.

The family of functions $M(t)$ is visualized on Fig. 3

The model $((3)-(4))$ for $\beta=20, \lambda=0.7, m=12, \alpha=1.5 t_{0}=0.763706$ is visualized on Fig. 4. From the nonlinear equation (6) and inequalities (7) we have: $d=0.092167, d_{l}=0.0646642, d_{r}=0.177086$.

The model $((3)-(4))$ for $\beta=30, \lambda=0.5, m=30, \alpha=1.5 t_{0}=0.887758$ is visualized on Fig. 5. From the nonlinear equation (6) and inequalities (7) we have: $d=0.0548628, d_{l}=0.031846, d_{r}=0.109768$.

For some comparisons between models $N$ (Eq. (1)) and $M$ (Eq. (3)) see Table 1. The applied comparisons show that the "saturation" using the $M$-model is faster. 


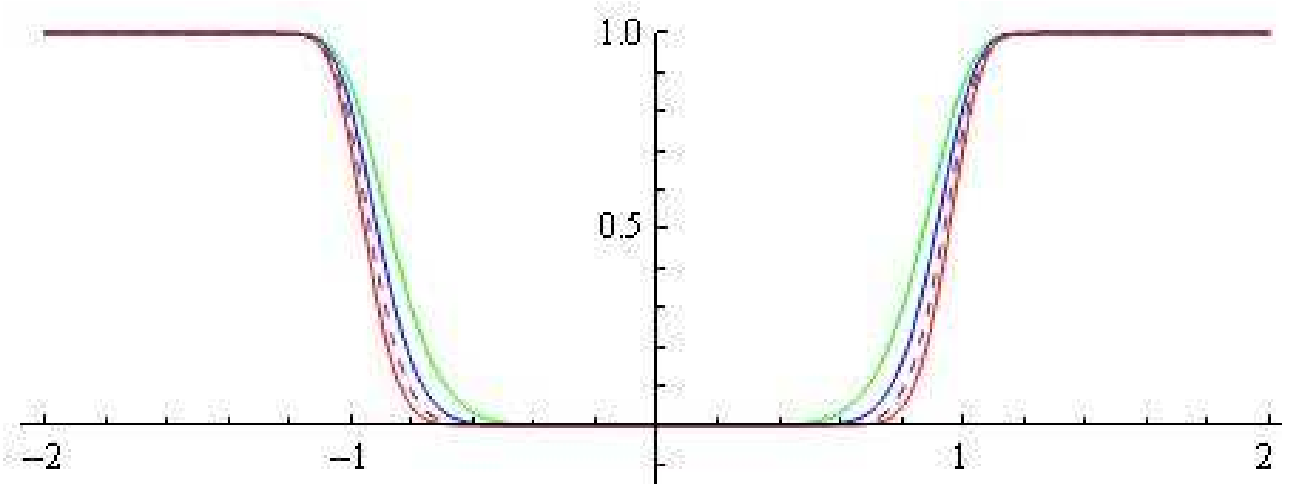

Figure 3: The family of functions $M(t)$ for $\alpha=1$ (green); $\alpha=1.5$ (blue); $\alpha=2$ (dashed); $\alpha=2.5$ (red); $(\beta=2, m=8$ and $\lambda=0.1$ are fixed).

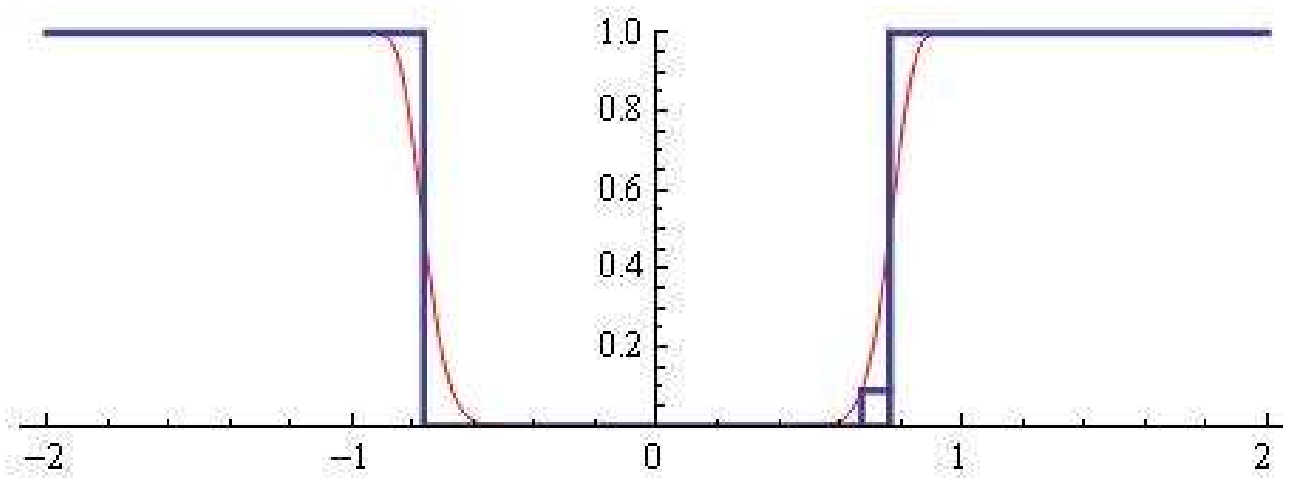

Figure 4: The model ((3)-(4)) for $\beta=20, \lambda=0.7, m=12, \alpha=1.5$ $t_{0}=0.763706 ; \mathrm{H}$-distance $d=0.092167, d_{l}=0.0646642, d_{r}=0.177086$.

\begin{tabular}{|l|l|l|l|l|l|}
\hline Model & $\beta$ & $\lambda$ & $m$ & $\alpha$ & Hausdorff Distance $-d$ \\
\hline $\mathrm{N}$ & 20 & 0.7 & 12 & 1 & 0.106819 \\
\hline $\mathrm{N}$ & 30 & 0.5 & 30 & 1 & 0.0663519 \\
\hline \hline $\mathrm{M}$ & 20 & 0.7 & 12 & 1.5 & 0.092167 \\
\hline $\mathrm{M}$ & 30 & 0.5 & 30 & 1.5 & 0.0548628 \\
\hline
\end{tabular}

Table 1. Some comparisons between models $N$ and $M$.

From the above examples, it can be seen that the proven estimates (see Theorem 2) for the value of the Hausdorff approximation are reliable when assessing the important characteristic - "saturation". 


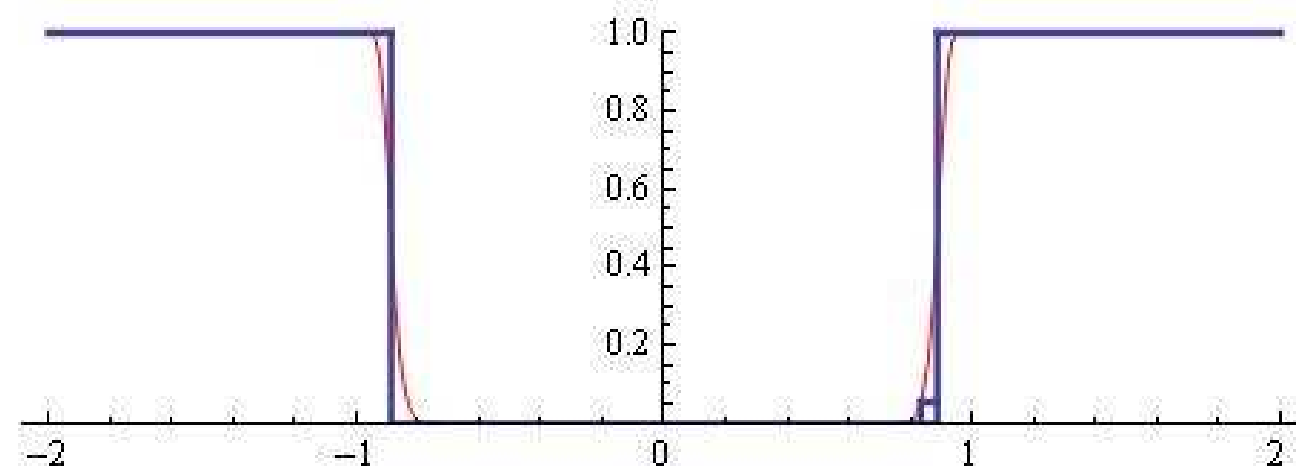

Figure 5: The model ((3)-(4)) for $\beta=30, \lambda=0.5, m=30, \alpha=1.5$ $t_{0}=0.887758 ; \mathrm{H}$-distance $d=0.0548628, d_{l}=0.031846, d_{r}=0.109768$.

\section{SOFTWARE MODULE FOR ANALYSIS WITHIN CAS MATHEMATICA}

We propose a software module (intellectual property) within the programming environment CAS Mathematica for the analysis of the considered family $M(t)$ of cumulative functions.

The module offers the following possibilities:

- generation of the impulse function under user defined values of the parameters $\lambda, m, \beta$ and $\alpha$;

- calculation of the H-distance $d$ between the function $\sigma^{* *}(t)$ and the function $M(t)$;

- generation of the emitting chart of antenna factor;

- software tools for animation and visualization.

After the substitution

$$
t=k l \cos \theta+a,
$$

where $k=\frac{2 \pi}{\lambda} ; \lambda$ is the wave length; $a$ is the phase difference; $\theta$ is the azimuthal angle and $l$ is the distance between the emitters ( $l=\frac{\lambda}{2}$ is fixed), the function $M(t)$ (or emitting chart of an antenna factor) can be rewritten in the form

$$
M(\theta)=\left(\frac{e^{\lambda e^{-\beta(\pi \cos \theta+a)^{m}}}-e^{\lambda}}{1-e^{\lambda}}\right)^{\alpha} .
$$

Typical emitting chart is visualized on Fig. 6. 


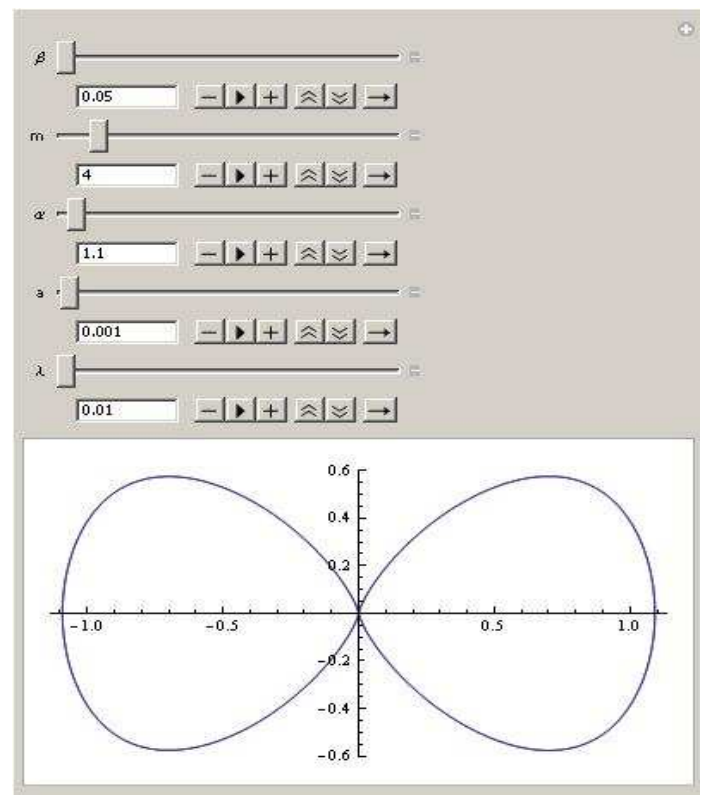

Figure 6: Typical emitting chart $(M(\theta))$ for $\beta=0.015 ; a=0.1 ; \lambda=0.15$; $m=4$.

\section{OPEN PROBLEMS AND CONCLUSIONS}

Of course, the question of the practical realization of the activation functions which are generated as emitting charts remains open.

The mathematical apparatus proposed in the article can be successfully used for imitation and simulation of such charts.

We will explicitly say that the results have independent significance in the study of issues related to impulse techniques [15].

Hausdorff approximation of some impulse functions and some modeling aspects in the field of antenna-feeder technique can be found in [16]-[29].

We hope that the results will be useful for a lot of specialists in this scientific area.

\section{ACKNOWLEDGMENTS}

This work has been supported by the project FP17-FMI-008 of Department for Scientific Research, Paisii Hilendarski University of Plovdiv. 


\section{REFERENCES}

[1] Z. Chen, A new two-parameter lifetime distribution with bathtub shape or increasing failure rate function, Stat. and Prob. Letters, 49 (2000), 155-161.

[2] Y. Chaubey, R. Zhang, An extension of Chen's family of survival distributions with bathtub shape or increasing hazard rate function, Comm. in Stat. - Theory and Methods, 44 (2015), 4049-4069.

[3] M. Xie, Y. Tang, T. Goh, A modified Weibull extension with bathtub - shaped failure rate function, Reliability Eng. and System Safety, 76 (2002), 279-285.

[4] M. Khan, A. Sharma, Generalized order statistics from Chen distribution and its characterization, J. of Stat. Appl. and Prob., 5 (2016), 123-128.

[5] S. Dey, D. Kumar, P. Ramos, F. Louzada, Exponentiated Chen distribution: Properties and Estimations, Comm. in Stat. - Simulation and Computation, (2017), 1-22.

[6] V. Cancho, F. Louzada, G. Barriga, The Poisson-exponential lifetime distribution, Comp. Stat. Data Anal., 55 (2011), 677-686.

[7] G. Rodrigues, F. Louzada, P. Ramos, Poisson-exponential distribution: different methods of estimation, J. of Appl. Stat., 45 (2018), no. 1, 128-144.

[8] F. Louzada, P. Ramos, P. Ferreira, Exponential-Poisson distribution: estimation and applications to rainfall and aircraft data with zero occurrence, Communication in Statistics-Simulation and Computation, (2018).

[9] N. Pavlov, A. Iliev, A. Rahnev, N. Kyurkchiev Some Software Reliability Models: Approximation and Modeling Aspects, LAP LAMBERT Academic Publishing, (2018), ISBN: 978-613-9-82805-0

[10] N. Pavlov, A. Iliev, A. Rahnev, N. Kyurkchiev Nonstandard Models in Debugging Theory (Part 2), LAP LAMBERT Academic Publishing, (2018), ISBN: 978-6139-87794-2.

[11] N. Kyurkchiev, A. Iliev, A Note on The "Saturation" of Poisson-Exponential Cumulative Function in Hausdorff Sense, Communications in Advanced Mathematical Sciences, (2018) (to appear).

[12] N. Kyurkchiev, A new class activation functions with application in the theory of impulse technics, Journal of Mathematical Sciences and Modelling, 1 (2018), no. 1, 15-20.

[13] O. Rahneva, H. Kiskinov, H. Melemov, M. Stieger, Some approximation Aspects for a new class cumulative distribution functions, Neural, Parallel, and Scientific Computations, 26 (2018), no. 2, 225-235, doi: 10.12732/npsc.v26i2.6 
[14] F. Hausdorff, Set Theory (2 ed.) (Chelsea Publ., New York, (1962 [1957]) (Republished by AMS-Chelsea (2005), ISBN: 978-0-821-83835-8.

[15] N. Kyurkchiev, A. Andreev, Approximation and Antenna and Filter Synthesis: Some Moduli in Programming Environment Mathematica, LAP LAMBERT Academic Publishing, Saarbrucken, (2014), ISBN 978-3-659-53322-8.

[16] N. Kyurkchiev, Bl. Sendov, Approximation of a class of functions by algebraic polynomials with respect to Hausdorff distance, Ann. Univ. Sofia, Fac. Math., 67 (1975), 573-579, (in Bulgarian).

[17] N. Kyurkchiev, S. Markov, On the Hausdorff distance between the Heaviside step function and Verhulst logistic function, J. Math. Chem., 54 (2016), no. 1, 109-119.

[18] A. Andreev, N. Kyurkchiev, Approximation of some impulse functions - implementation in programming environment MATHEMATICA, Proceedings of the 43 Spring Conference of the Union of Bulgarian Mathematicians, Borovetz, April 2-6, (2014), 111-117.

[19] N. Kyurkchiev, S. Markov, On the numerical approximation of the "cross" set, Ann. Univ. Sofia, Fac. Math., 66 (1974), 19-25, (in Bulgarian).

[20] N. Kyurkchiev, A. Andreev, Hausdorff approximation of functions different from zero at one point - implementation in programming environment MATHEMATICA, Serdica J. of Computing, 7 (2013), no. 2, 135-142.

[21] N. Kyurkchiev, A. Andreev, Synthesis of slot aerial grids with Hausdorff-type directive patterns - implementation in programming environment Mathematica, C.R. Acad. Bulg. Sci., 66 (2013), no. 11, 1521-1528.

[22] N. Kyurkchiev, Synthesis of Slot Aerial Grids with Hausdorff Type Directive Patterns, PhD Thesis, Department of Radio-Electronics, VMEI, Sofia (1979), (in Bulgarian).

[23] Bl. Sendov, H. Schinev, N. Kjurkchiev, Hausdorff-synthesis of aerial grids in scanning the directive diagram, Electropromishlenost $i$ Priboroostroene, 16 (1981), no. 5, 203-205, (in Bulgarian).

[24] H. Schinev, N. Kjurkchiev, M. Gachev, Experimental investigations of slot aerial grids with Hausdorff type directive patterns, it Electropromishlenost i Priboroostroene, 14 (1979), no. 6, 223-224, (in Bulgarian).

[25] H. Shinev, N. Kyurkchiev, M. Gachev, S. Markov, Application of a class of polynomials of best approximation to linear antenna array synthesis, Izv. VMEI Sofia, 34 (1975), no. 1, 1-6, (in Bulgarian).

[26] A. Golev, T. Djamiykov, N. Kyurkchiev, Sigmoidal functions in antenna-feeder technique, Int. J. of Pure and Appl. Math., 116 (2017), no. 4, 1081-1092. 
[27] N. Pavlov, A. Iliev, A. Rahnev, N. Kyurkchiev, On the extended Chen's and Pham's software reliability models. Some applications, Int. J. of Pure and Appl. Math., 118 (2018), no. 4, 1053-1067.

[28] S. Markov, N. Kyurkchiev, A. Iliev, A. Rahnev, On the approximation of the cut functions by hyper-log-logistic function, Neural, Parallel, and Scientific Computations, 26 (2018), no. 2, 169-182, doi: 10.12732/npsc.v26i2.3

[29] Q. Wang, Y. Zhang, Y. Guo, A novel heterogeneous training sample selection method on space-time adaptive processing, AIP Conference Proceedings, 1955, 040148 (2018), doi: 10.1063/1.5033812 